\title{
Numerical analysis of high strain rate failure of electro-magnetically loaded steel sheets
}

\author{
Borja Erice $^{1, \mathrm{a}}$ and Dirk Mohr ${ }^{1,2,3}$ \\ ${ }^{1}$ Solid Mechanics Laboratory (CNRS-UMR 7649), Department of Mechanics, École Polytechnique, Palaiseau, France \\ 2 Impact and Crashworthiness Lab, Department of Mechanical Engineering, Massachusetts Institute of Technology, \\ Cambridge MA, USA \\ ${ }^{3}$ Department of Mechanical and Process Engineering, ETH Zurich, Switzerland
}

\begin{abstract}
Electro-magnetic forces provide a potentially power full means in designing dynamic experiments with active control of the loading conditions. This article deals with the development of computational models to simulate the thermo-mechanical response of electro-magnetically loaded metallic structures. The model assumes linear electromagnetic constitutive equations and time-independent electric induction to estimate the Joule heating and the Lorentz forces. The latter are then taken into account when evaluating stress equilibrium. A thermo-visco-plastic model with Johnson-Cook type of temperature and strain rate dependence and combined Swift-Voce hardening is used to evaluate the material's thermo-mechanical response. As a first application, the model is used to analyse the effect of electro-magnetic loading on the ductility of advanced high strength steels.
\end{abstract}

\section{Introduction}

Modern equipment is able to discharge large capacitor energies in specifically designed coils generating electromagnetic (EM) forces in conductive workpieces in periods of time in the order of $0.1 \mathrm{~ms}$. Far from being spatially concentrated forces, their area of influence extends to that of the EM field generated in the coil. This might give an unprecedented level of tailoring and control on dynamic experiments. Additionally, a significant increase in ductility on conductive metals is reported with respect to conventional mechanical forming processes.

The objective of this numerical study is twofold. First, understand how the EM loading can affect to a dynamic deformation process and second dilucidate which are the underlying mechanisms behind the increase on ductility and delayed fracture. To do so, numerical simulations of electro-magnetically vs. mechanically loaded sheet metal plates were compared.

\section{Problem description}

Our analysis is concerned with the introduction of a hemispherical dome (of $50 \mathrm{~mm}$ radius) into an initially flat $1.4 \mathrm{~mm}$ thick steel sheet (see Fig. 1). Two different high speed loading scenarios are considered to achieve this: (1) electro-magnetic loading, and (2) mechanical impact loading. The goal is to compare these two techniques and to come up with an explanation of the well-known failure retardation in forming processes through electro-magnetic load application.

\footnotetext{
${ }^{a}$ Corresponding author: borja@lms.polytechnique.fr
}

\subsection{Electro-magnetically applied load}

The magnetic field is created by circulating a pulsed electric current through a coil that is positioned next to a conductor material. Figure 2 shows a serial RLC circuit that provides the required current, with $L_{\mathrm{RLC}}$ and $R_{\mathrm{RLC}}$ denoting the inductance and resistivity of the circuit, respectively. The current of intensity $i_{c}(t)$ circulating through the coil with a resistance $R_{\text {coil }}$ and an inductance $L_{\text {coil }}$ is generated by the sudden discharge of the energy stored in capacitor $C$. Assuming an equivalent $\bar{R}, \bar{L}, C$ circuit, the expression for the current intensity of the underdamped response circuit [1] reads

$$
i_{c}(t)=\frac{V_{0}}{\omega \bar{L}} \exp \left(-\frac{\bar{R}}{2 \bar{L}} t\right) \sin (\omega t), \omega=\sqrt{\frac{1}{\bar{L} C}-\left(\frac{\bar{R}}{2 \bar{L}}\right)^{2}}
$$

with $\omega$ denoting the natural frequency.

Spirally wounded coils are typically employed to apply the Lorentz forces as uniformly as possible to the workpiece [2]. The configuration adopted for the present numerical study is detailed in Fig. 1(a). A $5 \times 5 \mathrm{~mm}^{2}$ square cross-section coil with four turns is located underneath the steel specimen. The overall coil diameter is chosen such as that the coil covers the entire $100 \mathrm{~mm}$ diameter die. The current intensity $i_{c}(t)$ is imposed through the coil as indicated in red colour in Fig. 1(a). The direction of the prescribed current intensity is such that the Lorentz forces generated in the workpiece are oriented upwards, in the positive direction of the $\mathrm{z}$-axis. As a result, the workpiece is pushed into the receiving die. The die is assumed to be non-conductive, fully rigid and fixed in space. A blank holder is positioned underneath the plate to clamp the specimen. The current intensity is

This is an Open Access article distributed under the terms of the Creative Commons Attribution License 4.0, which permits unrestricted use, distribution, and reproduction in any medium, provided the original work is properly cited. 


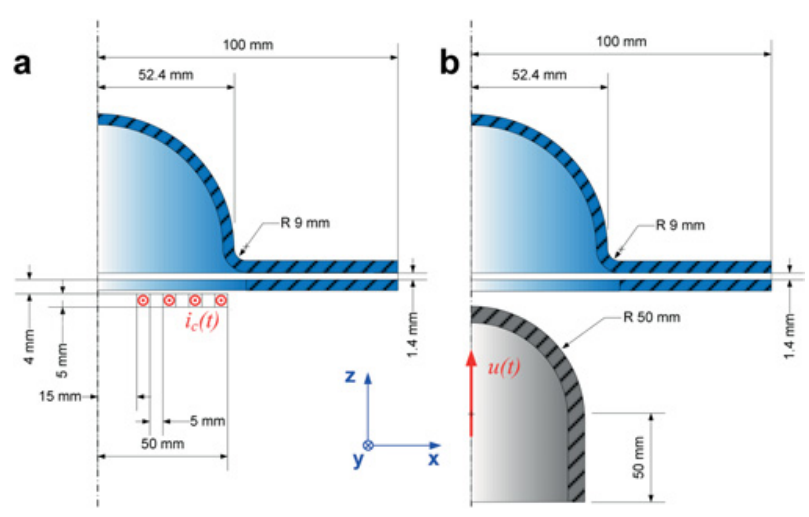

Figure 1. Schematic view of the EM (a) and mechanical (b) problems.

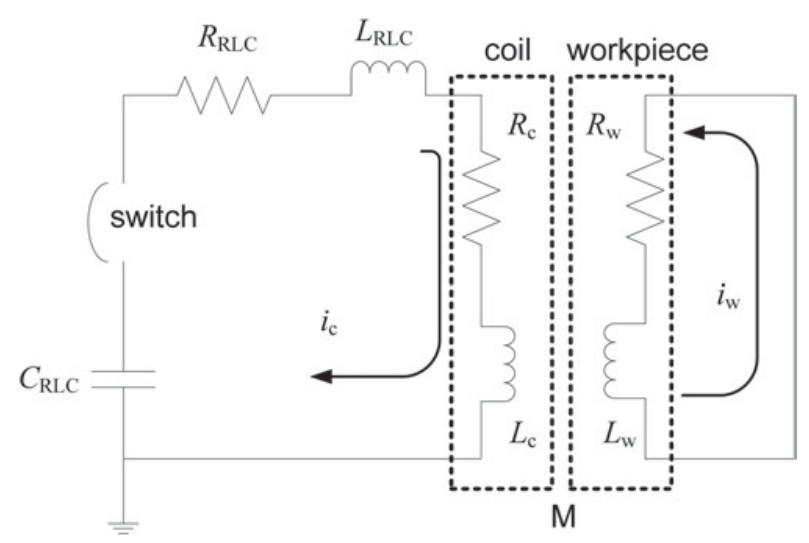

Figure 2. Simplified RLC circuit used in the electro-magnetic loading processes. $\mathrm{M}$ represents the mutual inductance.

calculated based Eq. (1) with an initial voltage of $V_{0}=$ $11 \mathrm{kV}$, a capacitance of $C=900 \mu \mathrm{F}$ and an inductance of $L=1 \mu \mathrm{H}$. The capacitor energy is released rapidly, generating a damped current intensity that is attenuated within about $0.3 \mathrm{~ms}$. The entire forming process will also be completed within this duration.

\subsection{Mechanically applied load}

In the mechanical process, the sheet is loaded through a hemispherical punch. The duration of a conventional mechanical sheet metal forming process is of the order of seconds, while the typical duration of the electro-magnetic process is about four orders of magnitude shorter. In our comparative study, we consider two mechanical loading scenarios: a slow case that is complete within $1 \mathrm{~s}$, and a fast case that completes the forming within $0.3 \mathrm{~ms}$. Moreover, to ensure the direct comparability, the punch velocity history in the fast process is chosen such that it matched the velocity of the dome apex in the electro-magnetic process.

\section{Thermo-mechanical constitutive model}

The mechanical constitutive response of the material presented here is based on the work by Mohr et al. [3], which subsequently was modified by Roth and Mohr [4] to take into account strain rate and thermal softening effects.
The model assumes the additive decomposition the strain tensor $\boldsymbol{\varepsilon}$ as:

$$
\boldsymbol{\varepsilon}=\boldsymbol{\varepsilon}^{e}+\boldsymbol{\varepsilon}^{p}
$$

with the elastic and plastic parts $\varepsilon^{e}$ and $\varepsilon^{p}$. The elastic strain and Cauchy stress tensors are related through Hooke's law as follows:

$$
\boldsymbol{\sigma}=\mathbf{C}:\left(\boldsymbol{\varepsilon}-\boldsymbol{\varepsilon}^{p}\right)
$$

where $\mathbf{C}$ is the positive-definite fourth-order symmetric tensor that contains the elastic modulus $E$ and the Poisson's ratio $v$,

$$
\mathbf{C}=\frac{v E}{(1+v)(1-2 v)} \boldsymbol{I} \otimes \boldsymbol{I}+\frac{E}{(1+v)} \mathbf{I}
$$

being $\boldsymbol{I}$ and $\mathbf{I}$ the identity second-order and fourth-order tensors, respectively.

The Hill 1948 orthotropic yield function [5] is adopted:

$$
\phi(\sigma, \alpha)=\bar{\sigma}_{\phi}(\sigma)-Y(\boldsymbol{\alpha}, \dot{\alpha})=0
$$

where $Y$ is a deformation resistance, $\alpha$ is a set of internal hardening variables, and $\dot{\alpha}=d \boldsymbol{\alpha} / d t$ their time derivative. $\bar{\sigma}_{\phi}$ is the yield function equivalent stress that can be expressed conveniently with a linear transformation of the stress tensor as follows [6]:

$$
\bar{\sigma}_{\phi}(\sigma)=\sqrt{\sigma: \mathbf{P}: \sigma}
$$

with the positive-definite fourth-order symmetric tensor,

$$
\mathbf{P}=\left[\begin{array}{ccccc}
1 & P_{12}-\left(1+P_{12}\right) & 0 & 0 & 0 \\
P_{22}-\left(P_{12}+P_{22}\right) & 0 & 0 & 0 \\
& 1+2 P_{12}+P_{22} & 0 & 0 & 0 \\
& & P_{33} & 0 & 0 \\
& \text { sym } & & 3 & 0 \\
& & & & 3
\end{array}\right] .
$$

The three independent components $P_{12}, P_{22}$ and $P_{33}$ can be expressed as a function of the uniaxial tension yield stresses in three directions with respect to the rolling direction, typically at 0,45 and 90 degrees and the equi-biaxial tension yield stress [3]. The yield function defines a von Mises yield surface in the case of isotropic yielding being $P_{12}=-0.5, P_{22}=1.0$ and $P_{33}=3.0$. A non-associated flow potential of the form,

$$
\bar{\sigma}_{\psi}(\sigma)=\sqrt{\sigma: \mathbf{G}: \sigma}
$$

has been chosen to represent the flow rule $\dot{\boldsymbol{\varepsilon}}^{p}=$ $\dot{\lambda}(\partial \psi / \partial \sigma)$. The choice of a non-associated flow rule (NAFR) for sheet metal forming is based on experimental observations. $\mathbf{G}$ is a positive-definite fourth-order symmetric tensor that has the same form as the $\mathbf{P}$. The three independent components $G_{12}, G_{22}$ and $G_{33}$ are defined as a function of the Lankford ratios corresponding to the same three directions used in the yield function [3]. 
The set of internal variables are the equivalent plastic strain, the equivalent plastic strain rate and the temperature; their evolution equations read:

$$
\dot{\bar{\varepsilon}}_{p}=\dot{\lambda} \frac{\bar{\sigma}_{\psi}}{\bar{\sigma}_{\phi}}, \ddot{\bar{\varepsilon}}_{p}=\frac{d \dot{\bar{\varepsilon}}_{p}}{d t}=\frac{d}{d t}\left(\dot{\lambda} \frac{\bar{\sigma}_{\psi}}{\bar{\sigma}_{\phi}}\right), \dot{T}=\dot{\lambda} \frac{\chi\left(\dot{\bar{\varepsilon}}_{p}\right)}{\rho C_{p}} \bar{\sigma}_{\psi}
$$

where $\rho$ is the mass density and $C_{p}$ is the specific heat. Typically, the temperature increase is assumed to be only due to adiabatic effects and the Taylor-Quinney coefficient $\chi$ is assumed to be constant and equal to 0.9. In an isothermal situation such a coefficient would be null. A smooth transition between isothermal and adiabatic conditions is proposed as [4]:

$$
\chi\left(\dot{\bar{\varepsilon}}_{p}\right)=\chi_{0}\left(\dot{\bar{\varepsilon}}_{p}-\dot{\varepsilon}_{0}\right)^{2}\left(3 \dot{\varepsilon}_{a}-2 \dot{\bar{\varepsilon}}_{p}-\dot{\varepsilon}_{0}\right) /\left(\dot{\varepsilon}_{a}-\dot{\varepsilon}_{0}\right)^{3}
$$

being $\dot{\varepsilon}_{0}$ and $\dot{\varepsilon}_{a}$ the limit strain rates for the isothermal and adiabatic domains, respectively.

Isotropic hardening is assumed accounting for strain hardening, strain rate hardening and the thermal softening has been considered independent [7-9]. Therefore, the constitutive response can be conveniently expressed in three separate terms that multiply each other as [4]:

$$
Y\left(\bar{\varepsilon}_{p}, \dot{\bar{\varepsilon}}_{p}, T\right)=Y_{\varepsilon}\left(\bar{\varepsilon}_{p}\right) Y_{\dot{\varepsilon}}\left(\dot{\bar{\varepsilon}}_{p}\right) Y_{T}(T) .
$$

The strain hardening is defined as a weighted linear combination of a Swift and Voce laws. It reads:

$$
\begin{aligned}
Y_{\varepsilon}= & \alpha\left\{A\left(\varepsilon_{0}+\bar{\varepsilon}_{p}\right)^{n}\right\}+(1-\alpha)\left\{\sigma_{0}+Q_{1}\right. \\
& {\left.\left[1-\exp \left(-C_{1} \bar{\varepsilon}_{p}\right)\right]\right\} }
\end{aligned}
$$

where $A, \varepsilon_{0}, n, \sigma_{0}, Q_{1}, C_{1}$ are material constants and $\alpha$ is the weighting coefficient. The strain rate hardening and the thermal softening have the same form as those defined by Johnson and Cook [9],

$$
Y_{\dot{\varepsilon}}=1+C \ln \left(\frac{\dot{\bar{\varepsilon}}_{p}}{\dot{\varepsilon}_{0}}\right), Y_{T}=1-\left(\frac{T-T_{r}}{T_{m}-T_{r}}\right)^{m}
$$

where $C$ and $m$ are material constants, $T_{r}$ and $T_{m}$ are the reference and melting temperatures respectively.

The Hosford-Coulomb failure criterion is a stress state dependent failure criterion. The stress state is characterized by the stress triaxiality $\eta$ and the Lode angle parameter $\bar{\theta}$. The expression of the equivalent plastic strain to failure is composed of three independent terms. The first term represents the stress-state-dependent failure criterion, while the second and the third are the expressions that introduce the strain rate and temperature effects defined as in [10]. The equivalent plastic strain to failure of the failure criterion is:

$$
\bar{\varepsilon}_{p}^{f}=\bar{\varepsilon}_{\eta, \bar{\theta}}^{f}(\eta, \bar{\theta}) \bar{\varepsilon}_{\dot{\varepsilon}}^{f}\left(\dot{\bar{\varepsilon}}_{p}\right) \bar{\varepsilon}_{T}^{f}(T) .
$$

The independent terms are defined as follows:

$$
\begin{aligned}
\bar{\varepsilon}_{\eta, \bar{\theta}}^{f}= & b(1+c)^{\frac{1}{n}}\left(\left\{\frac{\left(F_{1}^{a}+F_{2}^{a}+F_{3}^{a}\right)}{2}\right\}^{\frac{1}{a}}\right. \\
& \left.+c\left(2 \eta+f_{1}+f_{3}\right)\right)^{-\frac{1}{n}}
\end{aligned}
$$

$$
\begin{aligned}
& F_{1}=f_{1}-f_{2}, \quad F_{2}=f_{2}-f_{3}, \quad F_{3}=f_{1}-f_{3} \\
& f_{1}=\frac{2}{3} \cos \left[\frac{\pi}{6}(1-\bar{\theta})\right], \quad f_{2}=\frac{2}{3} \cos \left[\frac{\pi}{6}(3+\bar{\theta})\right] \\
& f_{3}=-\frac{2}{3} \cos \left[\frac{\pi}{6}(1+\bar{\theta})\right] \\
& \bar{\varepsilon}_{\dot{\varepsilon}}^{f}=1+D_{4} \ln \left(\frac{\dot{\bar{\varepsilon}}_{p}}{\dot{\varepsilon}_{0}}\right), \bar{\varepsilon}_{T}^{f}=1+D_{5} \frac{T-T_{r}}{T_{m}-T_{r}}
\end{aligned}
$$

where $a, b, c, D_{4}$ and $D_{5}$ are material constants. The damage indicator evolution law (failure for $D=1$ ) is defined as

$$
\dot{D}=\frac{1}{\bar{\varepsilon}_{p}^{f}\left(\eta, \bar{\theta}, \dot{\bar{\varepsilon}}_{p}, T\right)} \dot{\bar{\varepsilon}}_{p} .
$$

It should be noted that since the model is uncoupled, i.e. no softening due to damage evolution; the damage indicator is not an internal variable of the plasticity model. The time discretized equations were integrated in an elastic predictor-plastic corrector-type set of equations and the return to the yield surface was executed with a cutting plane algorithm. The calibration procedure for the DP590 steel can be found in [4] (see Table 1).

\section{Numerical set-up}

Due to its symmetry conditions only one fourth of the geometry was modeled. The discretization was performed using eight-node reduced-integration solid elements with stiffness-based hourglass control. The die and the punch were modelled as non-conductive rigid bodies. The die was fixed in space, while a displacement $u(t)$ was prescribed to the punch. Six elements over the thickness were used for the work piece, whereas for the die and punch, since they were modelled as rigid bodies, only one element was used through the thickness. The in-plane element size of the plate and dies was $1 \mathrm{~mm}$. The end of the plate was considered to be free of constrains. The only constraint comes from the blanket holder that prevents any specimen motion in z-direction (see Table 1).

A Boundary Element Method (BEM) is employed to circumvent the meshing of the surrounding air $[11,12]$. The electromagnetic and the mechanical solvers are coupled through the addition of Lorentz forces to the stress equilibrium equations in form of nodal forces. Following the developer's recommendation [11], the chosen time step of the electromagnetic solver was about 100 times the mechanical time step. Thus, the electromagnetic solver will be active every 100 mechanical increments. The wires were assumed to be made of pure electrical quality copper with $E=117 \mathrm{GPa}, v=0.3$ and a mass $\rho=8920 \mathrm{~kg} / \mathrm{m}^{3}$. The wire turns were modelled as individual parts. The same current intensity was prescribed in all coils. Therefore, the current displacement effect between turns was considered negligible. In the electromagnetic forming all the materials involved were considered to exhibit their free-space magnetic permeability. The electrical conductivity of the die and coils were considered to be zero and $5.96 \times 10^{7} \mathrm{~S} / \mathrm{m}$ respectively. The electrical conductivity for the DP 590 steel is contained in Table 1. 
Table 1. Material constants for the DP590 steel.

\begin{tabular}{|c|c|c|c|c|c|c|c|}
\hline$\rho\left(\frac{\mathrm{kg}}{\mathrm{m}^{3}}\right)$ & $C_{p}\left(\frac{\mathrm{J}}{\mathrm{kg}^{\circ} \mathrm{C}}\right)$ & $E(\mathrm{GPa})$ & $v$ & $\chi_{0}$ & $\dot{\varepsilon}_{a}\left(\mathrm{~s}^{-1}\right)$ & \multicolumn{2}{|c|}{$\Sigma_{0}\left(\frac{S}{m}\right)$} \\
\hline 7850.0 & 420.0 & 210.0 & 0.3 & 0.9 & 1.379 & \multicolumn{2}{|c|}{$1.5 \times 10^{6}$} \\
\hline$P_{12}$ & $P_{22}$ & $P_{33}$ & $G_{12}$ & \multicolumn{2}{|c|}{$G_{22}$} & \multicolumn{2}{|c|}{$G_{33}$} \\
\hline-0.5000 & 1.0000 & 3.0000 & -0.4946 & & .9318 & \multicolumn{2}{|c|}{2.4653} \\
\hline$A(\mathrm{MPa})$ & $\varepsilon_{0}$ & $n$ & $\sigma_{0}(\mathrm{MPa})$ & & $(\mathrm{MPa})$ & $C_{1}$ & $\alpha$ \\
\hline 1031.00 & 0.00128 & 0.199 & 350.00 & & 24.25 & 25.92 & 0.64 \\
\hline$C$ & $\dot{\varepsilon}_{0}\left(\mathrm{~s}^{-1}\right)$ & $m$ & $T_{0}\left({ }^{\circ} \mathrm{C}\right)$ & & $\Gamma_{r}\left({ }^{\circ} \mathrm{C}\right)$ & \multicolumn{2}{|c|}{$T_{m}\left({ }^{\circ} \mathrm{C}\right)$} \\
\hline 0.0136 & 0.001164 & 0.921 & 25.0 & & 25.0 & \multicolumn{2}{|c|}{1400.6} \\
\hline$a$ & $b$ & $c$ & $n$ & & $D_{4}$ & \multicolumn{2}{|c|}{$D_{5}$} \\
\hline 1.970 & 0.820 & 0.000 & 0.199 & & 0.025 & \multicolumn{2}{|c|}{0.000} \\
\hline
\end{tabular}
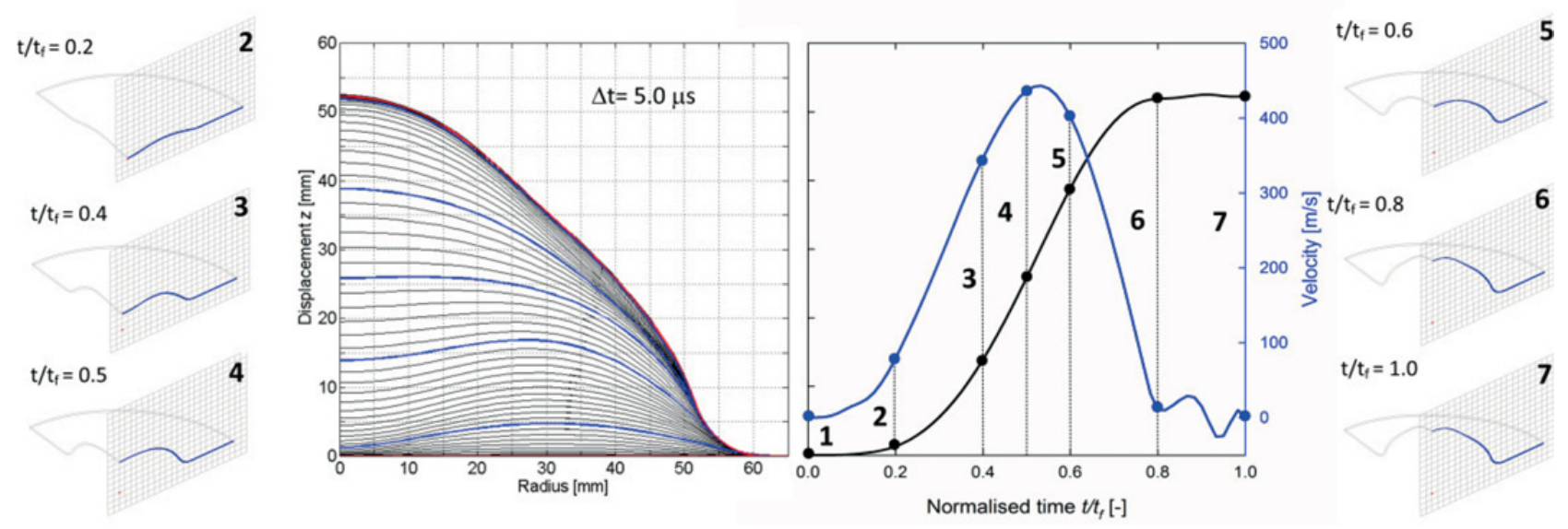

Figure 3. Evolution of the cross-section upper surface depicted in blue in the lateral pictures (left). Displacement and velocity vs. normalized time of the point located on the center of the plate (right).

\section{Results and discussion}

Firstly, we analyze the EM forming process and the characteristic steps leading to the final sheet shape. The right hand side plot in Fig. 3 shows the displacement (black) and velocity (blue) histories of the point located in the center of the upper surface of the sheet. The time has been normalized by the total duration of the simulations $\left(t_{f}\right)$. The plot is marked with seven solid points that correspond to $t / t_{f}=[0.0,0.2,0.4,0.5,0.6,0.8,1.0]$. For each of these positions, a snapshot of the deformed geometry was taken (see left and right of Fig. 3). To enhance the visualization of the progressive deformation of the sheet, a cross-section cut along one of the specimen symmetry planes is illustrated in blue over a grid formed by $5 \times 5 \mathrm{~mm}$ squares. Additionally, on the left hand side of Fig. 3 the evolution with time of such cross-section upper line is plotted every $5 \mu$ s time intervals. The red curves correspond to the initial and final states, $t / t_{f}=[0.0,1.0]$, whereas the blue curves correspond to the intermediate steps, $t / t_{f}=[0.2,0.4,0.5,0.6,0.8]$.

In the initial stage of the plate forming, i.e. from steps one to two, the Lorentz forces generated on the sheet metal plate were concentrated in the area of influence of the coils. As a result, there was a lack of magnetic pressure on the center of the specimen. Its effect on the deformed shape vanished quickly after about $10 \mu$ s (beyond point two in Fig. 3).

From there onwards, the velocity was then transferred from the part driven by Lorentz forces towards the center of the plate. Moreover, the inertia of the workpiece appeared to be driving the deformation process beyond point four where the velocity was about to reach its maximum (see Fig. 3 (right)). In the next stage, from point four to five, the displacement of the area close to the center kept displacing at the same pace as it did it the stage before. Conversely, there was a significant loss of velocity that indicated the commencing of the progressive slow-down of the plate (see Fig. 3 (right)). During the subsequent stage, from step five to six, it suffered a dramatic drop on velocity completing the slow-down process and attaining virtually its maximum displacement. In the last simulated stage, between points six and seven, there were nearly null forces that could affect the deformation of the workpiece. Hence, the center point practically maintained its displacement, while the velocity oscillated around zero.

Figure 4 shows the upper dome profile evolution of the two mechanically-loaded cases (slow and fast) compared to the EM-loaded case. Note that the time increment for each one of the black solid curves plotted in the slow case is the $20 \mathrm{~ms}$ and green curves coincide with the time the first fracture appears. In the slow case, displayed in Fig. 4(a), the plate deformation was driven by the force applied with the punch. That means that the parts of the plate that were not in contact with it were dragged immediately by the zones that were actually in contact. The first zone of the workpiece to be contacted by the punch was the center of it. Consequently, it can be seen how it was the first zone to be lifted and then the rest of the plate followed. The way in which the plate was being 

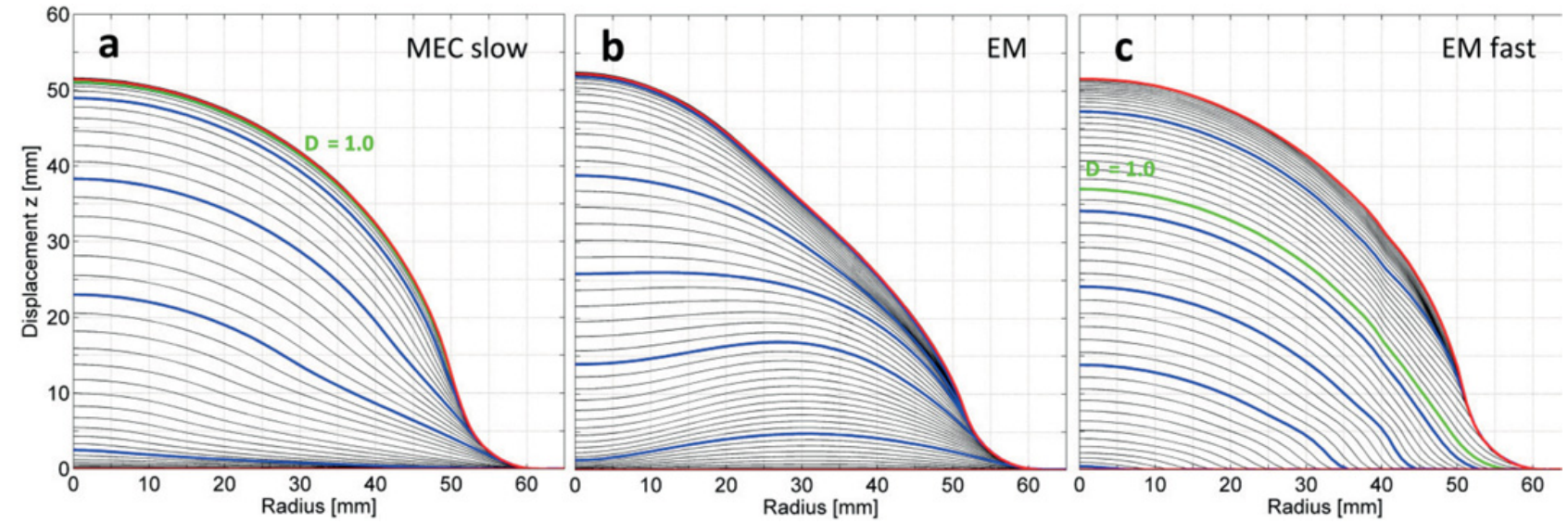

Figure 4. Evolution with time of the slow (a), EM (b) and fast (c) cases.

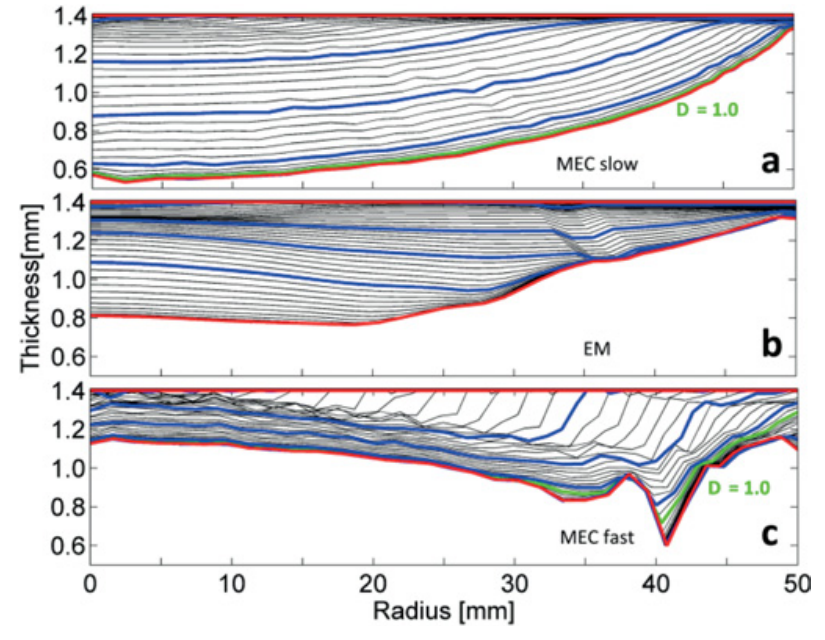

Figure 5. Evolution of the thickness with time of the slow (a), EM (b) and fast (c) cases.

formed made the thinning to be localized within a narrow plastic zone. Therefore, the workpiece necked rapidly in the center area causing its fracture before it reached the pre-established maximum displacement. The progressive thinning and necking on the center area can be seen in Fig. 5(a).

The fast case resembles an impact problem, where the punch acts like a guided projectile and the sheet as the target. The only difference here is that the projectile has a prescribed motion in contrast with a typical impact problem. In Fig. 4(c) we observe that even though the principle applied to move the plate was the same as that in the slow case, the plate did not deform in the same way. The center of the workpiece "saw" the contact but the rest of it still did not noticed the impact yet. Figure 5(c) depicts perhaps more clearly such phenomenon. There was a stress wave that travelled from the first point where the punch impacted, the center, towards the end of the plate. This relieved such part from strains. There was a moment when the plate did not move by the effect of the punch but by the effect of the inertia. Recall that a similar effect occurred in the in the EM case. The inertia then dragged the central part of the plate, which was not in contact with the punch anymore, necking severely the zone that was highly strained shortly before.

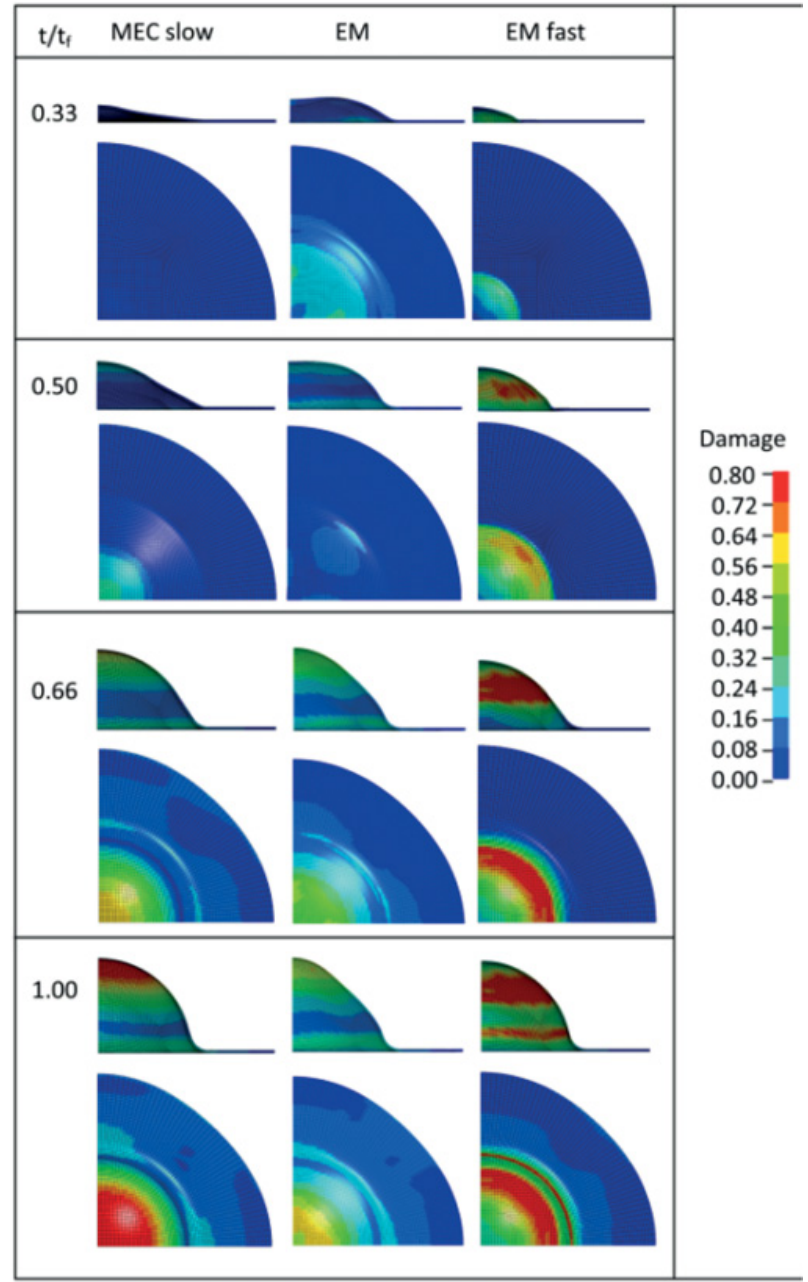

Figure 6. Damage contours of the numerical simulations carried out for the slow (a), EM (b) and fast (c) cases.

The differences on the damage accumulation of the three study cases, slow, EM and fast, are represented in Fig. 6. The differences between the slow and fast cases are clearly distinguishable here. On one hand, the progressive plastic strain accumulation, and therefore damage accumulation, in the central zone, can be observed in the slow case. On the other hand, the absence of such 
accumulation in the fast case central zone can be clearly seen. In fact, the plastic strain accumulation, as explained before, occurs away from the specimen center. In any case, both slow and fast cases presented a severe thinning and subsequent necking as can be seen in Fig. 5. Conversely to these cases, the more uniform force distribution of the EM case, allowed the plate to thin relatively uniformly and without necking, revealing EM loaded process as the less harmful for the material in terms of fracture. The manner in which the load was applied in this case allowed the strains to distribute more uniformly over the plate and extend the load-applying area not solely to the center, but to far more distance than the mechanically-driven forces allowed. On top of that, the load-applying period was small enough to benefit from strain rate and thermal softening effects which also increase the material's ductility. Indeed, Fig. 6 shows how the damage accumulation in the plate is far from the levels of the mechanically loaded cases.

\section{Conclusions}

This paper presents a computational model predicting the high strain rate failure of electro-magnetically loaded structures. In addition to the electro-magnetic and mechanical solvers, it makes use of a newly-developed rate- and temperature dependent user material subroutine. In particular, the Hill yield surface with a non-associated flow rule was used in conjunction with a JohnsonCook type of isotropic rate-dependent hardening law. The stress-state, strain-rate and temperature dependent Hosford-Coulomb fracture initiation model was used to evaluate the damage accumulation on the deformation processes.

In a first application, the modeling approach was used to analyze the apparent ductility increase in electromagnetic forming through comparison with simulations with conventional mechanical loading. The analysis showed that the load application in the mechanical cases caused pronounced strain localization within the deforming sheet, whereas significantly more uniform strain distributions are observed for electromagnetic loading. The delayed failure in electromagnetic forming is thus mainly attributed to the increased strain field uniformity, whereas the increase in ductility due to strain rate and temperature effects is seen as secondary.

The first author is grateful for the partial financial support through the French National Research Agency (Grant ANR-11-BS090008, LOTERIE).

\section{References}

[1] A. Alonso Rodriguez and A. Valli, Eddy Current Approximation of Maxwell Equations. MS\&A, ed. T.Y. Hou, et al. Vol. 4. (2010): Springer.

[2] V. Psyk, D. Risch, B.L. Kinsey, A.E. Tekkaya, and M. Kleiner, Electromagnetic forming-A review. Journal of Materials Processing Technology, (2011). 211(5): p. 787-829.

[3] D. Mohr, M. Dunand, and K.-H. Kim, Evaluation of associated and non-associated quadratic plasticity models for advanced high strength steel sheets under multi-axial loading. International Journal of Plasticity, (2010). 26(7): p. 939-956.

[4] C.C. Roth and D. Mohr, Effect of Strain Rate on Ductile Fracture Initiation in Advanced High Strength Steel Sheets: Experiments and Modeling. International Journal of Plasticity (2014) (In press).

[5] R. Hill, A theory of the yielding and plastic flow of anisotropic metals. roceedings of the Royal Society of London. Series A, Mathematical and Physical Sciences, (1948). 193(1033): p. 281-297.

[6] M.A. Crisfield, Non-linear Finite Element Analysis of Solids and Structures, Volume 2: Advanced Topics. (1997), London: John Wiley \& Sons.

[7] T. Børvik, Hopperstad, O.S., Berstad, T., Langseth, M., A computational model of viscoplasticity and ductile damage for impact and penetration. European Journal of Mechanics - A/Solids, (2001). 20: p. 685-712.

[8] S. Chocron, B. Erice, and C.E. Anderson, A new plasticity and failure model for ballistic application. International Journal of Impact Engineering, (2011). 38(8-9): p. 755-764.

[9] G.R. Johnson and W.H. Cook. A Constitutive Model and Data for Metals Subjected to Large Strains, High Strain Rates and High Temperatures. in 7th International Symposium on Ballistics. (1983). The Hague.

[10] G.R. Johnson and W.H. Cook, Fracture characteristics of three metals subjected to various strains, strain rates, temperatures and pressures. Engineering Fracture Mechanics, (1985). 21: p. 31-48.

[11] P. L'Eplanettenier and I. Çaldichoury, EM Theory Manual, Livermore Software Technology Corporation (LSTC). (2012). 40.

[12] LSTC, LS-DYNA KEYWORD USER'S MANUAL Version 971 R7.0. (2013), Livermore, California: Livermore Software Technology Corporation. 